\title{
Capsule Commentary on Wolff, et al., A Look at Person- and Family- Centered Care Among Older Adults: Results from a National Survey
}

\author{
Carla Maria Perissinotto, MD, MHS and Louise C. Walter, MD ${ }^{1,2}$ \\ 'University of California, San Francisco, CA, USA; ${ }^{2}$ San Francisco VA Medical Center, San Francisco, CA, USA.
}

$\mathrm{J}$ Gen Intern Med 30(10): 1536

DOI: $10.1007 / \mathrm{s} 11606-015-3408-1$

(c) Society of General Internal Medicine 2015

$\mathrm{P}$ erson- and family-centered care involves weighing potential benefits and burdens of medical treatments according to the values and preferences of patients and their families. ${ }^{1}$ Such decision-making is especially crucial as people age and develop comorbidities, which increase opportunities for more medical treatments and the susceptibility to treatment burdens. ${ }^{2}$ A key finding In the article by Wolff and Boyd ${ }^{3}$ was that nearly $40 \%$ of older adults reported treatment burden - that healthcare activities were difficult, didn't get done, or were too much to do. Burden was even more likely for older adults in poor health and/or those with limited literacy.

Person- and family-centered care aims to reduce the burden of medical interventions by prioritizing care that leads to outcomes most valued by an individual and/or family. Therefore, it is important to ask about an older person's goals and preferences regarding quality of life, longevity and functional status in order to align medical recommendations with these goals. The answer to "What are your goals?" may reveal that a certain burdensome treatment is not the best choice. Second, it is important to ask about the burdens of the proposed interventions and treatment plans in older adults. This includes broadening the definition of burden to include not just medical morbidity and mortality, but potential "social burdens" as well. Social burdens encompass a wide array of factors, including financial concerns, transportation difficulties, and labeling people as ill. Medical care does not stand as an isolated issue in people's lives. Lastly, it is important to ask about a person's approach to medical decisions within their broader social context. The article by Wolff and Boyd ${ }^{3}$ highlights the considerable variation among older adults as to whether they prefer making decisions about their care independently, jointly with others, or by completely delegating decision-making to someone else.

Asking questions about treatment goals, burdens and decision-making preferences is an important step towards providing person- and family-centered care, in contrast to care focused on what is best for an organ or disease. Ultimately, having this broader view has the potential to reduce the medical footprint in older people's lives.

Conflict of Interest: The authors have no conflicts of interest.

Corresponding Author: Carla Maria Perissinotto, MD, MHS; University of California, 3333 California St. Suite 380, San Francisco, CA 94143, USA (e-mail: Carla.Perissinotto@ucsf.edu).

\section{REFERENCES}

1. Gallacher K, Morrison D, Jani B, et al. Uncovering treatment burden as a key concept for stroke care: a systematic review of qualitative research. PLoS Med. 2013;10:e1001473.

2. Patient-centered care for older adults with multiple chronic conditions: a stepwise approach from the American Geriatrics Society: American Geriatrics Society Expert Panel on the Care of Older Adults with Multimorbidity. J Am Geriatr Soc. 2012;60(10):1957-68. doi:10.1111/j.1532-5415.2012.04187.x.

3. Wolff JL, Boyd CM. A Look at Person- and Family-Centered Care Among Older Adults: Results from a National Survey. J Gen Intern Med. doi:10. 1007/s11606-015-3359-6. 